\title{
ENTROPY RIGIDITY OF SYMMETRIC SPACES WITHOUT FOCAL POINTS
}

\author{
FRANÇOIS LEDRAPPIER AND LIN SHU \\ To Werner Ballmann for his 61st birthday
}

Abstract. We characterize symmetric spaces without focal points by the equality case of general inequalities between geometric quantities.

\section{INTRODUCTION}

Let $(M, g)$ be a closed connected Riemannian manifold and $\pi:(\widetilde{M}, \widetilde{g}) \rightarrow$ $(M, g)$ its universal cover endowed with the lifted Riemannian metric. We denote $p(t, x, y), t \in \mathbb{R}_{+}, x, y \in \widetilde{M}$ as the heat kernel on $\widetilde{M}$, the fundamental solution of the heat equation $\frac{\partial u}{\partial t}=\Delta u$ on $\widetilde{M}$, where $\Delta=\operatorname{Div} \nabla$ is the Laplacian on $\widetilde{M}$. Since we have a compact quotient, all the following limits exist as $t \rightarrow \infty$ and are independent of $x \in \widetilde{M}$ :

$$
\begin{aligned}
\lambda_{0} & =\inf _{f \in C_{c}^{2}(\widetilde{M})} \frac{\int|\nabla f|^{2}}{\int|f|^{2}}=\lim _{t}-\frac{1}{t} \ln p(t, x, x), \\
\ell & =\lim _{t} \frac{1}{t} \int d(x, y) p(t, x, y) d \operatorname{Vol}(y), \\
h & =\lim _{t}-\frac{1}{t} \int p(t, x, y) \ln p(t, x, y) d \operatorname{Vol}(y), \\
v & =\lim _{t} \frac{1}{t} \ln \operatorname{Vol} B_{\widetilde{M}}(x, t),
\end{aligned}
$$

where $B_{\widetilde{M}}(x, t)$ is the ball of radius $t$ centered at $x$ in $\widetilde{M}$ and Vol is the Riemannian volume on $\widetilde{M}$.

All these numbers are nonnegative. Recall that $\lambda_{0}$ is the bottom of the spectrum of the Laplacian, $\ell$ the linear drift, $h$ the stochastic entropy and $v$ the volume entropy. There is the following relation:

$$
4 \lambda_{0} \stackrel{(a)}{\leq} h \stackrel{(b)}{\leq} \ell v \stackrel{(c)}{\leq} v^{2} .
$$

See [25] for (a) and [16] for (b). Inequality (c) is shown in 28, as a corollary of (b) and (1.2):

$$
\ell^{2} \leq h
$$

In this paper, we are interested in the characterization of the locally symmetric property of $M$ by the equality case of the above inequalities for manifolds without focal points. Recall that a Riemannian manifold $M$ is said to have no focal points

Received by the editors May 4, 2012 and, in revised form, November 8, 2012.

2010 Mathematics Subject Classification. Primary 53C24, 53C20.

Key words and phrases. Volume entropy, rank one manifolds. 
if for any imbedded open geodesic segment $\gamma:(-a, a) \mapsto M$ (where $0<a \leq \infty)$, the restriction of the exponential map on the normal bundle of $\gamma$ is everywhere nonsingular. Hence any manifold of nonpositive curvature has no focal points. The reverse is not true since there exist manifolds without focal points but with sectional curvatures of both signs ([17]). If $M$ is a locally symmetric space without focal points, it must have nonpositive curvature ([18, Theorem 3.1]). Note that for symmetric spaces of nonpositive curvature, $4 \lambda_{0}=v^{2}$ ([21]; cf. [5, Appendice C]), and hence all five numbers $4 \lambda_{0}, \ell^{2}, h, \ell v, v^{2}$ coincide by (1.1), (1.2) above and are positive unless $(\widetilde{M}, g)$ is $\left(\mathbb{R}^{n}\right.$, Eucl.). Our result is a partial converse:

Theorem 1.1. Let $(M, g)$ be a compact connected Riemannian manifold without focal points. With the above notation, all the following equalities are equivalent to the locally symmetric property of $M$ :

i) $4 \lambda_{0}=v^{2}$;

ii) $h=\ell^{2}$ or $v^{2}$;

iii) $\ell=v$.

As recalled in 28, Theorem 1.1 is known in the negative curvature case and follows from [20, 7], 6], 11] and [25. The other possible converses are delicate: even for negatively curved manifolds, in dimension greater than two, it is not known whether $h=\ell v$ holds only for locally symmetric spaces. This is equivalent to a conjecture of Sullivan (see 27] for a discussion). Sullivan's conjecture holds for surfaces of negative curvature ([25, 22]). In the negative curvature case, $4 \lambda_{0}=h$ (and hence $4 \lambda_{0}=\ell v$ ) implies $M$ is locally symmetric, which follows from [26], 7], [6], and [11. In the no focal points case, whether $4 \lambda_{0}=h$ holds only for locally symmetric spaces may depend on a further study on the Martin boundary of $\widetilde{M}$ (cf. [1] for this notion). However, it would follow from the hypothetical $4 \lambda_{0} \leq \ell^{2}$ by ii) of Theorem 1.1 .

We assume henceforth that $(M, g)$ has no focal points. Given a geodesic $\gamma$ in $M$, Jacobi fields along $\gamma$ are vector fields $t \mapsto J(t) \in T_{\gamma(t)} M$ which describe infinitesimal variation of geodesics around $\gamma$. The rank of the geodesic $\gamma$ is the dimension of the space of Jacobi fields such that $t \mapsto\|J(t)\|$ is a constant function on $\mathbb{R}$. The rank of a geodesic $\gamma$ is at least one because of the trivial $t \mapsto \dot{\gamma}(t)$ which describes the variation by sliding the geodesic along itself. The rank of the manifold $M$ is the smallest rank of geodesics in $M$. Using the rank rigidity theorem for manifolds with no focal points from [32, we reduce in section 2 the proof of Theorem 1.1 to proving that if $(M, g)$ is rank one, the equality $\ell^{2}=h$ implies that $(\widetilde{M}, \widetilde{g})$ is a symmetric space. For this, we show in section 4 that the equality $\ell^{2}=h$ implies that $(\widetilde{M}, \widetilde{g})$ is asymptotically harmonic (see section 2.4 for its definition). This uses the solvability of the Dirichlet problem at infinity and the structure of harmonic measures of the stable foliation, which will be presented in section 3. Finally, it was recently observed by A. Zimmer ([33] that asymptotically harmonic universal covers of rank one manifolds are indeed symmetric spaces.

\section{Generalities and Reduction of Theorem 1.1}

We assume in the following that $(M, g)$ is a compact connected Riemannian manifold without focal points. Let $\widetilde{M}$ be the universal cover of $M$ with covering group $\Gamma=\pi_{1}(M)$. 
2.1. Geometric boundary. The notion of geometric boundary was introduced by Eberlein and O'Neill [8] for the nonpositive curvature case (see [3]). Consider the geodesics on $\widetilde{M}$. Two geodesic rays $\gamma_{1}$ and $\gamma_{2}$ of $\widetilde{M}$ are said to be asymptotic (or equivalent) if $\sup _{t>0} d\left(\gamma_{1}(t), \gamma_{2}(t)\right)<+\infty$. The set of equivalence classes $[\gamma]$, denoted by $\widetilde{M}(\infty)$, is called the geometric boundary of $\widetilde{M}$. We denote by $\widehat{M}$ the union $\widetilde{M} \cup \widetilde{M}(\infty)$.

Given $x \in \widetilde{M}$, for any geodesic ray $\gamma$ of $\widetilde{M}$, there exists a unique geodesic starting at $x$ which is asymptotic to $\gamma([31$, Proposition 3]). Hence for any $(x, \xi) \in$ $\widetilde{M} \times \widetilde{M}(\infty)$ there is a unique unit speed geodesic $\gamma_{x, \xi}$ satisfying $\gamma_{x, \xi}(0)=x$ and $\left[\gamma_{x, \xi}\right]=\xi$. Denote by $S_{x} \widetilde{M}$ the unit tangent space at $x$ to $\widetilde{M}$. The mapping $\pi_{x}^{-1}: \widetilde{M}(\infty) \mapsto S_{x} \widetilde{M}$ sending $\xi$ to $\dot{\gamma}_{x, \xi}(0)$ is a bijection between these two sets.

For $v, w \in S_{p} \widetilde{M}, p \in \widetilde{M}$, the angle $\angle_{p}(v, w)$ is the unique number $0 \leq \theta \leq \pi$ such that $\langle v, w\rangle=\cos \theta$. For $q \in \widehat{M}$ other than $p$, let $\gamma_{p, q}$ denote the unique unit speed geodesic starting at $p$ pointing at $q$. Given $v \in S_{p} \widetilde{M}$ and $0<\epsilon<\pi$, the set

$$
C(v, \epsilon):=\left\{q \in \widehat{M}: \quad \angle_{p}\left(v, \dot{\gamma}_{p, q}(0)\right)<\epsilon\right\}
$$

is called the cone of vertex $p$, axis $v$, and angle $\epsilon$ (cf. 8]). It was shown in 15 that there exists a canonical topology on $\widehat{M}$ so that for any $x \in \widetilde{M}$, the mapping $\pi_{x}$ is a homeomorphism between $S_{x} \widetilde{M}$ and $\widetilde{M}(\infty)$. The topology is called the "cone" topology in the sense that for $\xi \in \widetilde{M}(\infty)$, the truncated cones

$$
C(v, \epsilon, r)=C(v, \epsilon) \cap(\widehat{M} \backslash B(p, r))
$$

containing $\xi$, where $B(p, r)$ is the closed ball of radius $r$ about $p$, form a local basis at $\xi$.

We will identify $S \widetilde{M}$ with $\widetilde{M} \times \widetilde{M}(\infty)$ by $(x, v) \mapsto\left(x, \pi_{x} v\right)$. The action of $\Gamma$ on $M$ can be continuously extended to $\widetilde{M}(\infty)$. Hence the quotient $S M$ is identified with the quotient of $\widetilde{M} \times \widetilde{M}(\infty)$ under the diagonal action of $\Gamma$.

2.2. Stable Jacobi tensor. Let $\gamma$ be a geodesic in $(\widetilde{M}, \widetilde{g})$ and let $N(\gamma)$ be the normal bundle of $\gamma$ :

$$
N(\gamma):=\bigcup_{t \in \mathbb{R}} N_{t}(\gamma), \text { where } N_{t}(\gamma)=(\dot{\gamma}(t))^{\perp}=\left\{X \in T_{\gamma(t)} M:\langle X, \dot{\gamma}(t)\rangle=0\right\}
$$

A $(1,1)$ tensor along $\gamma$ is a family $V=\{V(t), t \in \mathbb{R}\}$, where $V(t)$ is an endomorphism of $N_{t}(\gamma)$ such that for any family $Y_{t}$ of parallel vectors along $\gamma$, the covariant derivative $V^{\prime}(t) Y_{t}:=\frac{D}{d t} V(t) Y_{t}$ exists.

We endow $N(\gamma)$ with Fermi orthonormal coordinates given by a parallel frame field along $\gamma$. A $(1,1)$ tensor along $\gamma$ is parallel if $V^{\prime}(t)=0$ for all $t$. It is then given by a constant matrix in Fermi coordinates. The curvature tensor $R$ induces a symmetric $(1,1)$ tensor along $\gamma$ by $R(t) X=R(X, \dot{\gamma}(t)) \dot{\gamma}(t)$. A $(1,1)$ tensor $V(t)$ along $\gamma$ is called a Jacobi tensor if it satisfies $V^{\prime \prime}+R V=0$. If $V(t)$ is a Jacobi tensor along $\gamma$, then $J(t):=V(t) Y_{t}$ is a Jacobi field for any parallel field $Y_{t}$.

For each $\mathbf{v}=(x, v) \in S \widetilde{M}$, let $\gamma_{\mathbf{v}}$ denote the unique geodesic starting from $x$ with speed $v$. A Jacobi tensor $V_{\mathbf{v}}$ defined for each $\gamma_{\mathbf{v}}$ is called continuous if the initial values $V_{\mathbf{v}}(0), V_{\mathbf{v}}^{\prime}(0)$ are continuous as $(1,1)$ tensors of the vector bundle $\mathbf{B}:=\{(\mathbf{v}, \mathbf{w}) \in S \widetilde{M} \times T \widetilde{M}: \quad \mathbf{w} \perp \mathbf{v}\}$ over $S \widetilde{M}$. 
Now for $\mathbf{v}=(x, v) \in S \widetilde{M}$, denote by $A_{\mathbf{v}}$ the Jacobi tensor along $\gamma_{\mathbf{v}}$ with initial condition $A_{\mathbf{v}}(0)=0$ and $A_{\mathbf{v}}^{\prime}(0)=I$ ( $I$ is the identity). For each $s>0$, let $S_{\mathbf{v}, s}$ be the Jacobi tensor with the boundary conditions $S_{\mathbf{v}, s}(0)=I$ and $S_{\mathbf{v}, s}(s)=0$. It can be shown (cf. [10]) that the limit $\lim _{s \rightarrow+\infty} S_{\mathbf{v}, s}=: S_{\mathbf{v}}$ exists and is given by

$$
S_{\mathbf{v}}(t)=A_{\mathbf{v}} \int_{t}^{+\infty}\left(A_{\mathbf{v}}^{*} A_{\mathbf{v}}\right)^{-1}(u) d u,
$$

where $A_{\mathbf{v}}^{*}$ is the transposed form of $A_{\mathbf{v}}$. The tensor $S_{\mathbf{v}}$ is called the stable tensor along the geodesic $\gamma_{\mathbf{v}}$. As a consequence of the uniform convergence of $S_{\mathbf{v}, s}^{\prime}(0)$ to $S_{\mathbf{v}}^{\prime}(0)\left(\left[\right.\right.$, Proposition 5]), one has by continuity of $S_{\mathbf{v}, s}^{\prime}(0)$ (with respect to $\mathbf{v}$ ) that the tensor $S_{\mathbf{v}}^{\prime}$ is continuous with respect to $\mathbf{v}([10$, Proposition 4$])$.

For each $\mathbf{v}=(x, v) \in S \widetilde{M}$, the vectors $\left(Y, S_{\mathbf{v}}^{\prime}(0) Y\right)$ describe variations of asymptotic geodesics and the subspace $E_{\mathbf{v}}^{s} \subset T_{\mathbf{v}} T \widetilde{M}$ they generate corresponds to $T W_{\mathbf{v}}^{s}$, where $W_{\mathbf{v}}^{s}$, the set of initial vectors of geodesics asymptotic to $\gamma_{\mathbf{v}}$, is identified with $\widetilde{M} \times \pi_{x}(v)$ in $\widetilde{M} \times \widetilde{M}(\infty)$. Recall that $S M$ is identified with the quotient of $\widetilde{M} \times \widetilde{M}(\infty)$ under the diagonal action of $\Gamma$. Clearly, for $\varphi \in \Gamma, \varphi\left(W_{\mathbf{v}}^{s}\right)=W_{D \varphi \mathbf{v}}^{s}$ so that the collection of $W_{\mathbf{v}}^{s}$ defines a foliation $\mathcal{W}^{s}$ on $S M$, the so-called stable foliation of $S M$. The leaves of the stable foliation $\mathcal{W}^{s}$ are a quotient of $\widetilde{M}$, and they are naturally endowed with the Riemannian metric induced from $\widetilde{g}$.

Similarly, by reversing the time in the construction of a stable tensor, one obtains the corresponding unstable tensor and hence the unstable subspaces and the unstable foliation.

2.3. Busemann functions. Fix $x_{0} \in \widetilde{M}$ as a reference point. For each $\xi \in \widetilde{M}(\infty)$, define a Busemann function at $\xi$ (cf. [9]) as follows. Let $\gamma_{x_{0}, \xi}$ be the unique unit speed geodesic starting at $x_{0}$ which is asymptotic to $\xi$. For each $s \geq 0$, defines the function

$$
b_{\xi, s}(x):=d\left(x, \gamma_{x_{0}, \xi}(s)\right)-s, \forall x \in \widetilde{M} .
$$

We have by triangle inequality that $b_{\xi, s}(x)$ are decreasing with $s$ and bounded absolutely from below by $-d\left(x_{0}, x\right)$. So the function

$$
b_{\xi}(x):=\lim _{s \rightarrow \infty} b_{\xi, s}(x), \forall x \in \widetilde{M},
$$

is well defined and is called the Busemann function at $\xi$. It was shown in [9] that the function $x \mapsto b_{\xi}(x)$ is of class $C^{2}$.

For each $\varphi \in \Gamma,(x, \xi) \in \widetilde{M} \times \widetilde{M}(\infty)$, we consider $b_{\varphi \xi}(\varphi x)$. The geodesics $\gamma_{x_{0}, \varphi \xi}$ and $\gamma_{\varphi x_{0}, \varphi \xi}$ are asymptotic. Hence the Busemann functions at $\varphi \xi$ using different reference points $x_{0}$ and $\varphi x_{0}$ only differ by a constant depending on $x_{0}, \varphi, \xi$ ([9, Proposition 3]), which is in fact given by $b_{\xi}\left(\varphi x_{0}\right)$. So we have

$$
\begin{aligned}
b_{\varphi \xi}(\varphi x) & :=\lim _{s \rightarrow+\infty}\left(d\left(\varphi x, \gamma_{x_{0}, \varphi \xi}(s)\right)-s\right) \\
& =\lim _{s \rightarrow+\infty}\left(d\left(\varphi x, \gamma_{\varphi x_{0}, \varphi \xi}(s)\right)-s\right)+b_{\xi}\left(\varphi x_{0}\right) \\
& =\lim _{s \rightarrow+\infty}\left(d\left(x, \gamma_{x_{0}, \xi}(s)\right)-s\right)+b_{\xi}\left(\varphi x_{0}\right) \\
& =b_{\xi}(x)+b_{\xi}\left(\varphi x_{0}\right) .
\end{aligned}
$$

It follows that the function $\Delta_{x} b_{\xi}$ satisfies $\Delta_{\varphi x} b_{\varphi \xi}=\Delta_{x} b_{\xi}$ and therefore defines a function $B$ on $\Gamma \backslash(\widetilde{M} \times \widetilde{M}(\infty))=S M$, which is called the Laplacian of the Busemann function. 
For $\mathbf{v}=(x, v) \in S \widetilde{M}$, let $\xi=\left[\gamma_{\mathbf{v}}\right]$ and let $b_{\mathbf{v}}:=b_{x, \xi}$, where $b_{x, \xi}$ is the Busemann function at $\xi$ using $x$ as a reference point. It is true ([9]) that

$$
\nabla_{w}\left(\nabla b_{\mathbf{v}}\right)=-S_{\mathbf{v}}^{\prime}(0)(w) .
$$

Since the stable tensor $S_{\mathbf{v}}^{\prime}$ is continuous with respect to $\mathbf{v}$, we have that $\Delta_{x} b_{\mathbf{v}}=$ $-\operatorname{Tr} S_{\mathbf{v}}^{\prime}(0)$ also depends continuously on $v$. Note that $\Delta_{x} b_{\mathbf{v}}=\Delta_{x} b_{\xi}$; we have that $\Delta_{x} b_{\xi}$ depends continuously on $\xi$. Consequently the function $B$ is continuous on $S M$.

2.4. Proof of Theorem 1.1. We continue assuming that $(\widetilde{M}, \widetilde{g})$ has no focal points. By the Rank Rigidity Theorem (see [32]), $(\widetilde{M}, \widetilde{g})$ is of the form

$$
\left(\widetilde{M}_{0} \times \widetilde{M}_{1} \times \cdots \times \widetilde{M}_{j} \times \widetilde{M}_{j+1} \times \cdots \times \widetilde{M}_{k}, \widetilde{g}\right), 1
$$

where $\widetilde{g}$ is the product metric $\widetilde{g}^{2}=\left(\widetilde{g}_{0}\right)^{2}+\left(\widetilde{g}_{1}\right)^{2}+\cdots+\left(\widetilde{g}_{j}\right)^{2}+\left(\widetilde{g}_{j+1}\right)^{2}+\cdots+\left(\widetilde{g}_{k}\right)^{2}$, $\left(\widetilde{M}_{0}, \widetilde{g}_{0}\right)$ is Euclidean, $\left(\widetilde{M}_{i}, \widetilde{g}_{i}\right)$ is an irreducible symmetric space of rank at least two for $i=1, \cdots, j$ and a rank one manifold for $i=j+1, \cdots, k$. If the $\left(\widetilde{M}_{i}, \widetilde{g}_{i}\right), i=$ $j+1, \cdots, k$, are all symmetric spaces of rank one, then $(\widetilde{M}, \widetilde{g})$ is a symmetric space. Moreover, in that case, all inequalities in (1.1) are equalities: this is the case for irreducible symmetric spaces (all numbers are 0 for Euclidean space; for the other spaces, note that locally a symmetric space without focal points must have nonpositive curvature ([18, Theorem 3.1]) and $4 \lambda_{0}$ and $v^{2}$ are classically known to coincide for a locally symmetric space with nonpositive curvature (cf. [5, Appendice $\mathrm{C}]$ )) and we have

$$
4 \lambda_{0}(\widetilde{M})=\sum_{i} 4 \lambda_{0}\left(\widetilde{M}_{i}\right), \quad v^{2}(\widetilde{M})=\sum_{i} v^{2}\left(\widetilde{M}_{i}\right) .
$$

To prove Theorem 1.1, it suffices to prove that if $\ell^{2}=h$, all $\widetilde{M}_{i}$ in the decomposition are symmetric spaces. This is already true for $i=0,1, \cdots, j$. It remains to show that $\left(\widetilde{M}_{i}, \widetilde{g}_{i}\right)$ are symmetric spaces for $i=j+1, \cdots, k$. Note that each one of the spaces $\left(\widetilde{M}_{i}, \widetilde{g}_{i}\right)$ admits a cocompact discrete group of isometries (see [23, Theorem 3.3] using the corresponding theorems from [32]). This shows that the linear drifts $\ell_{i}$ and the stochastic entropies $h_{i}$ exist for each one of the spaces $\left(\widetilde{M}_{i}, \widetilde{g}_{i}\right)$. Moreover, we clearly have

$$
\ell^{2}=\sum_{i} \ell_{i}^{2}, \quad h=\sum h_{i}
$$

Therefore Theorem 1.1 follows from

Theorem 2.1. Assume $(M, g)$ is a closed connected rank one manifold without focal points and that $\ell^{2}=h$. Then $(\widetilde{M}, \widetilde{g})$ is a symmetric space.

Let $(M, g)$ be a closed connected Riemannian manifold without focal points as before. Its universal cover $(\widetilde{M}, \widetilde{g})$ is said to be asymptotically harmonic if $B$, the Laplacian of the Busemann function, is constant on $S M$. In that case, we have by [33. Theorem 1.2] that $(M, g)$ is either flat or the geodesic flow on $S M$ is Anosov. The latter case, as was observed by Knieper [24, Theorem 3.6], actually implies that $M$ is a rank one locally symmetric space. (Indeed, let $M$ be as above with $\widetilde{M}$ being asymptotically harmonic. If the geodesic flow on $S M$ is Anosov, then it is true by P. Foulon and F. Labourie [11] that the stable and unstable distribution

\footnotetext{
${ }^{1}$ With a clear convention for the cases when $\operatorname{Dim} \widetilde{M}_{0}=0, j=0$ or $k=j$.
} 
$E^{s}$ and $E^{u}$ of the geodesic flow are $C^{\infty}$. Hence the result of Y. Benoist, P. Foulon and F. Labourie 7] applies and gives that the geodesic flow of $(M, g)$ is smoothly conjugate to the geodesic flow of a locally symmetric space $\left(M_{0}, g_{0}\right)$ of negative curvature. Note that in the no focal points case, the volume entropy and the topological entropy of the geodesic flow coincide [12. Thus one can use G. Besson, G. Courtois and S. Gallot's rigidity theorem ([6]) to conclude that the two spaces $(M, g)$ and $\left(M_{0}, g_{0}\right)$ are isometric.) In summary, we have

Proposition 2.2 ([33, Theorem 1.2]). Assume $(M, g)$ is a closed connected rank one manifold without focal points such that $(\widetilde{M}, \widetilde{g})$ is asymptotically harmonic. Then $(\widetilde{M}, \widetilde{g})$ is a symmetric space.

Therefore, Theorem 2.1 directly follows from Proposition 2.2 and

Proposition 2.3. Assume $(M, g)$ is a closed connected rank one manifold without focal points and that $\ell^{2}=h$. Then $(\widetilde{M}, \widetilde{g})$ is asymptotically harmonic.

\section{HARMonic MeAsure For the Stable Foliation}

We consider the stable foliation $\mathcal{W}:=\mathcal{W}^{s}$ of subsection 2.2. Recall that the leaves are endowed with a natural Riemannian metric. We write $\Delta^{\mathcal{W}}$ for the associated Laplace operator on functions which are of class $C^{2}$ along the leaves of $\mathcal{W}$. A probability measure $m$ on $S M$ is called harmonic if it satisfies, for any $C^{2}$ function $f$,

$$
\int_{S M} \Delta^{\mathcal{W}} f d m=0
$$

Our main result in this section is:

Theorem 3.1. Let $(M, g)$ be a closed connected rank one manifold without focal points and $\mathcal{W}$ the stable foliation on $S M$ endowed with the natural metric as above. Then, there is only one harmonic probability measure $m$ and the support of $m$ is the whole space $S M$.

A main step in the proof of Theorem 3.1 is to identify the lift of $m$ in $\widetilde{M} \times \widetilde{M}(\infty)$ locally as $d x \times d m_{x}$, where $m_{x}$ is the hitting probability at $\widetilde{M}(\infty)$ of the Brownian motion on $\widetilde{M}$ starting at $x$ and $d x$ is proportional to the Riemannian volume on $\widetilde{M}$. We adopt Ballmann's approach $([2,3])$ to use the Lyons-Sullivan procedure (30]) of discretizing the Brownian motion (starting at $x$ ) to a random walk on $\Gamma$ and show $m_{x}$ is indeed the unique stationary measure of the corresponding random process on $\Gamma$. The argument involves a random walk on $\Gamma$, the solvability of the Dirichlet problem at infinity and divergence properties of geodesics for manifolds without focal points and is divided into seven steps for clarity.

3.1. Discretization of Brownian motion. Fix $x_{0} \in \widetilde{M}$. The discretization procedure of Lyons and Sullivan ([30]) associates to the Brownian motion on $\widetilde{M}$ a probability measure $\nu$ on $\Gamma$ with $\nu(\varphi)>0$ for all $\varphi \in \Gamma$ such that any bounded harmonic function $h$ on $\widetilde{M}$ satisfies

$$
h\left(x_{0}\right)=\sum_{\varphi \in \Gamma} h\left(\varphi x_{0}\right) \nu(\varphi) .
$$


Consider the random walk on $\Gamma$ defined by $\nu$ with the transition probability of $\nu\left(\varphi^{-1} \widetilde{\varphi}\right)$ from $\varphi \in \Gamma$ to $\widetilde{\varphi} \in \Gamma$. For given $\varphi_{1}, \cdots, \varphi_{k}$, the probability $\mathbb{Q}$ that a sequence $\left\{\varphi_{n}\right\}$ begins with $\varphi_{1}, \cdots, \varphi_{k}$ is defined to be

$$
\nu\left(\varphi_{1}\right) \nu\left(\varphi_{1}^{-1} \varphi_{2}\right) \cdots \nu\left(\varphi_{k-1}^{-1} \varphi_{k}\right) .
$$

The random walk on $\Gamma$ generated by $\nu$ is a good approximation of Brownian motion on $\widetilde{M}$ starting at $x_{0}$ in the following sense:

Proposition 3.2 ([30, Theorem 6]; cf. [2, Sec. 4]). Let $W$ be the space of all continuous paths $c:(0,+\infty) \rightarrow \widetilde{M}$ and $\Omega$ the space of all sequences of heads and tails. There is a probability measure $\overline{\mathbb{P}}$ on $W \times \Omega$ with the following properties:

i) The natural projection from $W \times \Omega$ to $W$ maps $\overline{\mathbb{P}}$ to $\mathbb{P}$, the probability measure on $W$ associated to the Brownian motion starting at $x_{0}$.

ii) There is a map $W \times \Omega \rightarrow \Gamma^{\mathbb{N}},(c, \omega) \rightarrow\left\{\varphi_{n}(c, \omega)\right\}$, which maps $\overline{\mathbb{P}}$ onto the probability measure $\mathbb{Q}$.

iii) There is an increasing sequence of stopping times $T_{n}$ on $W \times \Omega$ and a positive constant $\delta<1$ such that

$$
\overline{\mathbb{P}}\left[\max _{T_{n}<t<T_{n+1}} d\left(c_{\omega}(t), \varphi_{n}(c, \omega)\right)>k\right] \leq \delta^{k}, \forall k>0 .
$$

3.2. Stationary measure of the random walk on $\Gamma$. Let $\nu$ be the LyonsSullivan measure on $\Gamma$ corresponding to $x_{0}$. Given a probability measure $\mu$ on $\widetilde{M}(\infty)$, define the convolution $\nu * \mu$ by letting

$$
\int_{\widetilde{M}(\infty)} f(\xi) d(\nu * \mu)(\xi)=\sum_{\varphi \in \Gamma}\left(\int_{\widetilde{M}(\infty)} f(\varphi \xi) d \mu(\xi)\right) \nu(\varphi)
$$

where $f$ is any bounded measurable function on $\widetilde{M}(\infty)$. The measure $\mu$ is called stationary (with respect to $\nu$ ) if

$$
\nu * \mu=\mu .
$$

Stationary measures with respect to $\nu$ always exist and are not supported on points ([3. p. 56]). For the uniqueness of the harmonic measure for the stable foliation, we first show that the hitting probability of the random walk on $\Gamma$ defined by $\nu$ is the unique stationary measure on $\widetilde{M}(\infty)$ with respect to $\nu$ and then identify this measure with the hitting probability of Brownian motion starting at $x_{0}$.

Theorem 3.3. Let $(M, g)$ be a closed connected rank one manifold without focal points. Let $\nu$ be a Lyons-Sullivan measure on $\Gamma$ as above. Then for the $\mathbb{Q}$-almost all sequence $\left\{\varphi_{n}\right\}$ in $\Gamma^{\mathbb{N}}$, the sequence $\left\{\varphi_{n} x\right\}, x \in \widetilde{M}$, tends to a limit in $\widetilde{M}(\infty)$. The hitting probability is given by the unique stationary measure on $\widetilde{M}(\infty)$ with respect to $\nu$.

The proof of Theorem 3.3 is the same as in [3, Theorem 4.11] once we show that the induced random walk on $\Gamma$ is transient and the Dirichlet problem for $\Gamma$ is solvable (see section 3.5 for the definition). In the following, we present successively some properties of hyperbolic points for $\Gamma$, the transient property of the random walk on $\Gamma$, and finally the solvability of the Dirichlet problem for the random walk on $\Gamma$. 
3.3. Hyperbolic points at infinity. A point $p \in \widetilde{M}(\infty)$ is called hyperbolic ([4]) if for any $q \neq p$ in $\widetilde{M}(\infty)$, there exists a rank one geodesic joining $q$ to $p$. A geodesic $\gamma$ of $\widetilde{M}$ is called an axis if there exists a $\varphi \in \Gamma$ and $a \in \mathbb{R}$ with $\varphi(\gamma(t))=\gamma(t+a)$ for all $t$. The endpoints of any rank one axial geodesic are hyperbolic ([32, Theorem 6.11]). On the other hand, we have by [32, Proposition 6.12] that for any pair of neighborhoods $U, V$ of the endpoints of a rank one geodesic at $\widetilde{M}(\infty)$, there exists a rank one axis with two endpoints in $U, V$, respectively. Note that the geodesic flow is topologically transitive for a rank one manifold without focal points ([19]). We have by the above argument that

Lemma 3.4. Let $(M, g)$ be a closed connected rank one manifold without focal points. The set of hyperbolic points is dense in $\widetilde{M}(\infty)$.

For hyperbolic points at infinity, we also have the following two lemmas from 32]:

Lemma 3.5 ([32, Lemma 6.18]). Let $p, q$ be the distinct points in $\widetilde{M}(\infty)$ with $p$ hyperbolic and suppose $U_{p}, U_{q}$ are neighborhoods of $p$ and $q$, respectively. Then there exists an isometry $\varphi \in \Gamma$ with

$$
\varphi\left(\widehat{M} \backslash U_{q}\right) \subset U_{p}, \quad \varphi^{-1}\left(\widehat{M} \backslash U_{p}\right) \subset U_{q} .
$$

Lemma 3.6 ([32, Lemma 6.19]). Let $p \in \widetilde{M}(\infty)$ be hyperbolic, $U^{*} \subset \widehat{M}$ a neighborhood of $p$, and $x \in \widetilde{M}$. Then there exists a neighborhood $U \subset \widehat{M}$ of $p$ such that if $\left\{\varphi_{n}\right\}$ is a sequence of isometries with $\varphi_{n}(x) \rightarrow x^{*} \in \widetilde{M}(\infty) \backslash U^{*}$, then

$$
\sup _{u \in U} \angle_{\varphi_{n}(x)}(x, u) \rightarrow 0, \text { as } n \rightarrow \infty,
$$

where $\angle_{a}(b, c)$ denotes the angle between the unit tangent vectors at a of the geodesics $\gamma_{a, b}$ and $\gamma_{a, c}$.

3.4. Transient random walk on $\Gamma$. If $\Gamma$ is not amenable, then it is true by Furstenberg [13, p. 212] that the random walk on $\Gamma$ generated by $\nu$ is transient, i.e. $d\left(x, \varphi_{n} x\right) \rightarrow \infty$ for $\mathbb{P}$ almost any sequence $\left\{\varphi_{n}\right\} \subset \Gamma$ (cf. [3, p. 58]). We show

Lemma 3.7. The covering group $\Gamma$ of a closed connected rank one manifold without focal points contains a free subgroup and hence is not amenable.

Proof. By Lemma 3.4, we can choose two neighborhoods $U_{i}, i=1,2$, of two hyperbolic points at $\widetilde{M}(\infty)$ so that each contains some additional point besides the hyperbolic point and they satisfy

$$
U_{1} \cap U_{2}=\varnothing, U_{1} \cup U_{2} \neq \widetilde{M}(\infty) .
$$

Then by Lemma 3.5, there exist isometries $\varphi_{1}$ and $\varphi_{2} \in \Gamma$ with

$$
\varphi_{1}\left(\widetilde{M}(\infty) \backslash U_{1}\right) \subset U_{1}, \quad \varphi_{2}\left(\widetilde{M}(\infty) \backslash U_{2}\right) \subset U_{2} .
$$

Hence for any $\xi \in \widetilde{M}(\infty) \backslash\left(U_{1} \cup U_{2}\right)$ and any nontrivial word $\varphi$ in $\varphi_{1}$ and $\varphi_{2}$, we see that $\varphi(\xi)$ belongs to $U_{i}$ if $\varphi$ begins with $\varphi_{i}$, and so $\varphi \not \equiv$ id. Consequently, $\varphi_{1}$ and $\varphi_{2}$ generate a free subgroup of $\Gamma$ and $\Gamma$ is nonamenable as desired. 
3.5. Dirichlet problem at infinity for $\Gamma$. A function $h: \Gamma \rightarrow \mathbb{R}$ is called $\nu$ harmonic if

$$
h(\varphi)=\sum_{\psi \in \Gamma} h(\varphi \psi) \nu(\psi), \text { for any } \varphi \in \Gamma .
$$

Let $\mu$ be a $\nu$-stationary measure. For any bounded and measurable function $f$ on $\widetilde{M}(\infty)$, define $h_{f}$ on $\Gamma$ by letting

$$
h_{f}(\varphi)=\int_{\widetilde{M}(\infty)} f(\varphi \xi) d \mu(\xi) .
$$

Then $h_{f}$ is $\nu$ harmonic since $\mu$ is $\nu$-stationary. The Dirichlet problem for a random walk on $\Gamma$ generated by $\nu$ is solvable if for any bounded measurable function $f$ on $\widetilde{M}(\infty)$ and $\xi \in \widetilde{M}(\infty)$ a point of continuity for $f$, the function $h_{f}$ is continuous at $\xi$, i.e. if $\left\{\varphi_{n}\right\} \subset \Gamma$ is a sequence such that $\varphi_{n} x \rightarrow \xi$ (for one and hence for any $x \in \widetilde{M})$, then $h_{f}\left(\varphi_{n}\right) \rightarrow f(\xi)$.

Theorem 3.8. Let $(M, g)$ be a closed connected rank one manifold without focal points. Let $\nu$ be a Lyons-Sullivan measure on $\Gamma$. Then the Dirichlet problem at infinity for the random walk on $\Gamma$ generated by $\nu$ is solvable. Consequently, if $f: \widetilde{M}(\infty) \rightarrow \mathbb{R}$ is continuous, then $h_{f}$ is the unique $\nu$ harmonic function on $\Gamma$ extending continuously to $f$ at infinity.

Remark 3.9. The theorem holds true if we replace the Lyons-Sullivan measure by any probability measure on $\Gamma$ whose support generates $\Gamma$ as a semigroup.

The proof follows [3, Theorem 4.10]. But the key lemma Ballmann used (3. Lemma 4.9]) needs to be adjusted in the no focal points setting. We present the two parts as a whole for completeness.

Proof of Theorem 3.8. Let $\nu$ be a Lyons-Sullivan measure on $\Gamma$ and let $\mu$ be a $\nu$ stationary measure. Suppose the Dirichlet problem is not solvable. Then there is a bounded measurable function $f$ and a point $\xi \in \widetilde{M}(\infty)$ of continuity for $f$ such that there is $\left\{\phi_{n}\right\} \subset \Gamma$ with

$$
\phi_{n}\left(x_{0}\right) \rightarrow \xi \text {, but } h_{f}\left(\phi_{n}\right) \nrightarrow \rightarrow f(\xi) .
$$

We may assume without loss of generality that $f(\xi)=0$. Let $\left\{\varphi_{n}\right\} \subset \Gamma$ be such that $\lim _{n \rightarrow+\infty}\left|h_{f}\left(\varphi_{n}\right)\right|$ exists and is maximal along all such sequences in (3.2). Denote this by

$$
\widetilde{\delta}:=\lim _{n \rightarrow+\infty}\left|h_{f}\left(\varphi_{n}\right)\right| .
$$

To draw a contradiction, it suffices to show there exist $\varphi \in \Gamma$ and $\left\{n_{k}\right\} \subset \mathbb{N}$ such that

$$
\left|h_{f}\left(\varphi_{n_{k}} \varphi\right)\right| \leq \frac{1}{2} \widetilde{\delta}, \text { for } k \text { large. }
$$

Indeed, consider the $l$-th convolution $\nu^{l}$ of $\nu$ defined inductively by letting $\nu^{0}$ be the Dirac measure at the neutral element of $\Gamma$ and

$$
\nu^{l}(\widetilde{\phi})=\sum_{\psi \in \Gamma} \nu^{l-1}(\psi) \nu\left(\psi^{-1} \widetilde{\phi}\right), \quad l \geq 1 .
$$

It is easy to see that $\mu$ is also stationary with respect to $\nu^{\ell}$ and $h_{f}$ satisfies

$$
h_{f}\left(\varphi_{n_{k}}\right)=\sum_{\psi \in \Gamma} h_{f}\left(\varphi_{n_{k}} \psi\right) \nu^{\ell}(\psi) .
$$


Choose $\ell$ such that $\nu^{\ell}(\varphi)=: \alpha>0$. We can break $\Gamma$ into three subsets $\{\varphi\}, G, L$, where $G \subset \Gamma$ is finite so that $L=\Gamma \backslash(G \cup\{\varphi\})$ satisfies $\nu^{\ell}(L) \sup |f|<\alpha \widetilde{\delta} / 4$. Then we have by (3.4) that

$$
\begin{aligned}
\lim _{k \rightarrow+\infty}\left|h_{f}\left(\varphi_{n_{k}}\right)\right| & <\lim _{k \rightarrow+\infty}\left|\sum_{\psi \in G} h_{f}\left(\varphi_{n_{k}} \psi\right) \nu^{\ell}(\psi)\right|+\lim _{k \rightarrow+\infty}\left|h_{f}\left(\varphi_{n_{k}} \varphi\right)\right| \alpha+\frac{1}{4} \alpha \widetilde{\delta} \\
& \leq \nu^{\ell}(G) \cdot \widetilde{\delta}+\frac{3}{4} \alpha \cdot \widetilde{\delta} \quad\left(\text { recall that } \varphi_{n_{k}} \psi x_{0} \rightarrow \xi\right) \\
& \leq\left(1-\frac{\alpha}{4}\right) \widetilde{\delta} .
\end{aligned}
$$

This will contradict the choice of $\widetilde{\delta}$. with

For (3.3), we first choose by continuity of $f$ at $\xi$ a number $0<\epsilon<\frac{\widetilde{\delta}}{6 \max \{\sup |f|, 1\}}$

$$
|f(\eta)|<\frac{1}{3} \widetilde{\delta}, \text { for } \eta \in C_{x_{0}, \xi}(\epsilon),
$$

where $C_{x_{0}, \xi}(\epsilon)$ is the shadow at infinity of the cone of $x_{0}$ with axis $\dot{\gamma}_{x_{0}, \xi}(0)$ and angle $\epsilon$, i.e.

$$
C_{x_{0}, \xi}(\epsilon)=\left\{\eta \in \widetilde{M}(\infty): \quad \angle_{x_{0}}(\eta, \xi)<\epsilon\right\} .
$$

Next, we claim there exists a neighborhood $U$ of some point at $\widetilde{M}(\infty)$ with $\mu(U)<$ $\epsilon, \varphi \in \Gamma$ and a sequence $\left\{n_{k}\right\} \subset \mathbb{N}$ such that

$$
\varphi_{n_{k}} \varphi(\widetilde{M}(\infty) \backslash U) \subset C_{x_{0}, \xi}(\epsilon) .
$$

With this, we have by the definition of the function $h_{f}$ (see (3.1) ) that

$$
\begin{aligned}
\left|h_{f}\left(\varphi_{n_{k}} \varphi\right)\right| & \leq\left|\int_{\widetilde{M}(\infty) \backslash U} f\left(\varphi_{n_{k}} \varphi(\xi)\right) d \mu(\xi)\right|+\left|\int_{U} f\left(\varphi_{n_{k}} \varphi(\xi)\right) d \mu(\xi)\right| \\
& \leq \mu(\widetilde{M}(\infty) \backslash U) \cdot \frac{\widetilde{\delta}}{3}+\mu(U) \sup |f| \\
& <\frac{1}{2} \widetilde{\delta}
\end{aligned}
$$

What is left is to show (3.5). Let $\left\{n_{k}\right\} \subset \mathbb{N}$ be such that $\varphi_{n_{k}}^{-1} x_{0} \rightarrow \eta \in \widetilde{M}(\infty)$ as $k$ goes to infinity. Select two points $p, q \in \widetilde{M}(\infty)$ with $p$ hyperbolic and two disjoint neighborhoods $U_{p}^{*}, U_{q}$ in $\widehat{M}$ around $p, q$, respectively, so that their union is apart from $\{\eta\} \cup C_{x_{0}, \xi}(\epsilon)$. We can further require $\mu\left(U_{q}\right)<\epsilon$. Apply Lemma 3.6 to the hyperbolic point $p$. We obtain a neighborhood $U_{p} \subset U_{p}^{*}$ so that

$$
\sup _{u \in U_{p}} \angle_{\varphi_{n_{k}}^{-1} x_{0}}\left(x_{0}, u\right) \rightarrow 0 \text {, as } k \rightarrow+\infty .
$$

For neighborhoods $U_{p}, U_{q}$ of $p, q$, we apply Lemma 3.5 to obtain an isometry $\varphi \in \Gamma$ with

$$
\varphi\left(\widehat{M} \backslash U_{q}\right) \subset U_{p}, \varphi^{-1}\left(\widehat{M} \backslash U_{p}\right) \subset U_{q} .
$$

We show $U_{q}$ and $\varphi$ satisfy the requirement of (3.5). Let $y \in \widetilde{M}(\infty) \backslash U_{q}$. We have

$$
\begin{aligned}
\angle_{x_{0}}\left(\xi, \varphi_{n_{k}} \varphi(y)\right) & \leq \angle_{x_{0}}\left(\xi, \varphi_{n_{k}}\left(x_{0}\right)\right)+\angle_{x_{0}}\left(\varphi_{n_{k}}\left(x_{0}\right), \varphi_{n_{k}} \varphi(y)\right) \\
& =\angle_{x_{0}}\left(\xi, \varphi_{n_{k}}\left(x_{0}\right)\right)+\angle_{\varphi_{n_{k}}^{-1} x_{0}}\left(x_{0}, \varphi(y)\right) \\
& \leq \angle_{x_{0}}\left(\xi, \varphi_{n_{k}}\left(x_{0}\right)\right)+\sup _{u \in U_{p}} \angle_{\varphi_{n_{k}}^{-1} x_{0}}\left(x_{0}, u\right),
\end{aligned}
$$


where the first quantity goes to zero as $k$ goes to infinity since $\varphi_{n_{k}}\left(x_{0}\right) \rightarrow \xi$ (as $k \rightarrow \infty)$ and the second quantity goes to zero by (3.6). This shows (3.5) holds true for $k$ large.

3.6. Coincidence of two classes of hitting measures. To show the coincidence of the hitting probabilities at $\widetilde{M}(\infty)$ of the Brownian motion (starting at $x_{0}$ ) and $\nu$ the random walk on $\Gamma$, it suffices to show that for $\overline{\mathbb{P}}$ almost all trajectories $(c, \omega)$, $c_{\omega}(t)$ tend to the same limit as the sequence $\left\{\varphi_{n}(c, \omega)\right\}$. We need two more lemmas.

Lemma 3.10 ([10, Proposition 4]). Let $(M, g)$ be a closed connected Riemannian manifold without focal points. For any $x_{0} \in \widetilde{M}$, there exist positive numbers $\alpha$ and $T$ such that for $t \geq T$, the equality

$$
\|J(t)\| \geq \alpha t^{\frac{1}{2}}
$$

is satisfied uniformly by all Jacobi fields which vanish initially at $x_{0}$ and have initial covariant derivative of length 1.

Lemma 3.11 (see [2, Theorem 3.14]). There exists a number $\beta>0$ such that the random sequence $\left\{\varphi_{n}\right\}$ in $\Gamma$ satisfies

$$
\lim _{n \rightarrow \infty} \frac{1}{n} d\left(x_{0}, \varphi_{n}\left(x_{0}\right)\right)=\beta .
$$

Theorem 3.12. The random Brownian path converges at $\widetilde{M}(\infty)$. The hitting measure at $\widetilde{M}(\infty)$ coincides with the hitting measure at $\widetilde{M}(\infty)$ of the $\nu$-random walk on $\Gamma$.

Proof. First, we have for $\overline{\mathbb{P}}$ almost all trajectory $(c, \omega)$ that

$$
\varlimsup_{n \rightarrow \infty} \frac{1}{\sqrt{n}} \max _{T_{n}<t<T_{n+1}} d\left(c_{\omega}(t), \varphi_{n}(c, \omega) x_{0}\right)=0 .
$$

To see this, for any $\epsilon>0$ let

$$
A_{n, \epsilon}=\left\{(c, \omega): \max _{T_{n}<t<T_{n+1}} d\left(c_{\omega}(t), \varphi_{n}(c, \omega) x_{0}\right)>\epsilon \sqrt{n}\right\} .
$$

Then by iii) of Proposition 3.2, we have

$$
\sum_{n=0}^{+\infty} \overline{\mathbb{P}}\left(A_{n, \epsilon}\right) \leq \sum_{n=0}^{+\infty} \delta^{\epsilon \sqrt{n}},
$$

which is finite since $\sum_{n=0}^{+\infty} \delta^{\epsilon \sqrt{n}} \leq 1+2 \sum_{l=1}^{+\infty}(l+1) \delta^{\epsilon l}<+\infty$. So we have by the Borel-Cantelli lemma that $\bigcap_{m \in \mathbb{N}} \bigcup_{n \geq m} A_{n, \epsilon}$ has $\overline{\mathbb{P}}$ probability 0 and (3.7) follows since $\epsilon>0$ is arbitrary.

By Lemma 3.11, we have for $\overline{\mathbb{P}}$ almost all $(c, \omega) \in W \times \Omega$,

$$
\varliminf_{n \rightarrow \infty} \frac{1}{n} d\left(x_{0}, \varphi_{n}(c, \omega) x_{0}\right)=\beta>0,
$$

and hence we have by (3.7) that $d\left(x_{0}, c_{\omega}(t)\right) \rightarrow \infty$ as $t \rightarrow \infty$ as well.

Finally, Theorem 3.12 directly follows from

$$
\max _{T_{n}<t<T_{n+1}} \angle_{x_{0}}\left(c_{\omega}(t), \varphi_{n}(c, \omega) x_{0}\right) \rightarrow 0, \text { as } n \rightarrow \infty .
$$


Suppose (3.8) doesn't hold $\overline{\mathbb{P}}$-almost everywhere. For a set of $\overline{\mathbb{P}}$ positive measure of $(c, \omega)$, there is a $\theta>0$ and infinitely many $n$ such that

$$
\max _{T_{n}<t<T_{n+1}} \angle x_{0}\left(c_{\omega}(t), \varphi_{n}(c, \omega) x_{0}\right)>\theta .
$$

For any $0<\epsilon<\frac{1}{8} \beta$, for $\overline{\mathbb{P}}$ almost all $(c, \omega)$, there is $N>\max \{4 T / \beta, T\}$ (where $T$ is from Lemma 3.10) such that for $n>N$, we have

$$
\begin{aligned}
& d_{1}(n)=\max _{T_{n}<t<T_{n+1}} d\left(c_{\omega}(t), \varphi_{n}(c, \omega) x_{0}\right)<\epsilon \sqrt{n}, \\
& d_{2}(n)=d\left(x_{0}, \varphi_{n}(c, \omega)\left(x_{0}\right)\right)>\frac{1}{2} \beta n .
\end{aligned}
$$

Take $(c, \omega)$ so that (3.9), (3.10) and (3.11) hold true. For $n>N$ and $t \in\left(T_{n}, T_{n+1}\right)$, consider the geodesics $\gamma, \widetilde{\gamma}$ which start at $x_{0}$ and point at $c_{\omega}(t)$ and $\varphi_{n}(c, \omega) x_{0}$, respectively. On the one hand, we have by (3.10) and the triangle inequality that

$$
d\left(c_{\omega}(t), \varphi_{n}(c, \omega) x_{0}\right) \geq d\left(\gamma\left(d_{2}(n)\right), \widetilde{\gamma}\left(d_{2}(n)\right)\right)-\epsilon \sqrt{n} .
$$

On the other hand, since $d_{2}(n)>\beta n / 2>T$, we have by Lemma 3.10 and (3.9) that

$$
\begin{aligned}
d\left(\gamma\left(d_{2}(n)\right), \widetilde{\gamma}\left(d_{2}(n)\right)\right) & >\theta \alpha d_{2}(n)^{\frac{1}{2}} \\
& >\theta \alpha\left(\frac{1}{2} \beta n\right)^{\frac{1}{2}} \\
& >2 \epsilon \sqrt{n},
\end{aligned}
$$

if we choose $\epsilon<2^{-1} \theta \alpha\left(\frac{1}{2} \beta\right)^{\frac{1}{2}}$. This, together with (3.12), contradicts (3.10).

\subsection{Proof of Theorem 3.1.}

Proof. Let $m$ be a $\mathcal{W}$ harmonic probability measure on $S M$. Then, there is a unique $\Gamma$-invariant measure $\widetilde{m}$ on $S \widetilde{M}$ which coincides with $m$ locally. Seen as a measure on $\widetilde{M} \times \widetilde{M}(\infty)$, we claim that $\widetilde{m}$ is given, for any continuous $f$ with compact support, by

$$
\int f(x, \xi) d \widetilde{m}(x, \xi)=\int_{\widetilde{M}}\left(\int_{\widetilde{M}(\infty)} f(x, \xi) d m_{x}(\xi)\right) d x
$$

where $m_{x}$ is the hitting measure of Brownian motion at $\widetilde{M}(\infty)$ starting at $x$ and $d x$ is proportional to the Riemannian volume on $\widetilde{M}$.

First, there is a family of probability measures $x \mapsto m_{x}$ on $\widetilde{M}(\infty)$ satisfying (3.13) Such that, for all $g$ continuous on $\widetilde{M}(\infty), x \mapsto \int g(\xi) d m_{x}(\xi)$ is a harmonic function on $\widetilde{M}$. This follows from [14]: on the one hand, the measure $\widetilde{m}$ projects on $\widetilde{M}$ as a $\Gamma$-invariant measure satisfying $\int \Delta f d m=0$. The projection of $\widetilde{m}$ on $\widetilde{M}$ is proportional to volume and formula $(\underline{3.13})$ is the disintegration formula. On the other hand, if one projects $\widetilde{m}$ first on $\widetilde{M}(\infty)$, there is a probability measure $\mathbf{m}$ on $\widetilde{M}(\infty)$ such that

$$
\int f(x, \xi) d \widetilde{m}(x, \xi)=\int_{\widetilde{M}(\infty)}\left(\int_{\widetilde{M}} f(x, \xi) d m_{\xi}(d x)\right) d \mathbf{m}(\xi) .
$$

For $\mathbf{m}$-a.e. $\xi$, the measure $m_{\xi}$ is a harmonic measure on $\widetilde{M}$; therefore, for $\mathbf{m}$-a.e. $\xi$, there is a positive harmonic function $k_{\xi}(x)$ such that $m_{\xi}=k_{\xi}(x)$ Vol. Comparing 
the two expressions for $\int f d \widetilde{m}$, we see that, up to a normalizing constant, the measure $m_{x}$ is given by

$$
m_{x}(d \xi)=k_{\xi}(x) \mathbf{m}(d \xi) .
$$

We normalize $\mathbf{m}$ in such a way that $k_{\xi}\left(x_{0}\right)=1$ for almost all $\xi$. Then, $x \mapsto$ $\int_{\widetilde{M}(\infty)} g(\xi) d m_{x}(\xi)$ is indeed a harmonic function.

Next, for any $x_{0} \in \widetilde{M}$, let $\nu$ be the corresponding Lyons-Sullivan measure on $\Gamma$. Since for all $g$ continuous on $\widetilde{M}(\infty), x \mapsto m_{x}(g)$ is a harmonic function and $m_{\varphi x_{0}}=\varphi_{*} m_{x_{0}}$ for $\varphi \in \Gamma$, it follows that the measure $m_{x_{0}}$ is a stationary measure for $\nu$, i.e.

$$
m_{x_{0}}=\sum_{\varphi \in \Gamma} \varphi_{*} m_{x_{0}} \nu(\varphi)
$$

So we conclude from Theorem 3.3 and Theorem 3.12 that $m_{x_{0}}$ is the hitting probability at $\widetilde{M}(\infty)$ of the Brownian motion starting at $x_{0}$. Since $x_{0}$ was arbitrary in the above reasoning, we have the desired expression of the lift of $m$ as in (3.13).

Finally, we have by the solvability of the Dirichlet problem (Theorem 3.8) that each $m_{x}$ is fully supported on $\widetilde{M}(\infty)$. It follows that the unique $\mathcal{W}$ harmonic measure $m$ is fully supported on $S M$.

\section{A LineAR DRIFT CHARACTERIZATION OF LOCAL SYMMETRY}

For Theorem 2.1, it remains to show Proposition 2.3. Consider the action of $\Gamma$ on $\widehat{M}=\widetilde{M} \cup \widetilde{M}(\infty)$. Let $X_{M}$ be the quotient of the space $\widetilde{M} \times \widehat{M}$ by the diagonal action of $\Gamma$. To each $\xi \in \widehat{M}$ is associated the projection of $\widehat{W}_{\xi}$ of $\widetilde{M} \times\{\xi\}$ in $X_{M}$. As a subgroup of $\Gamma$, the stabilizer $\Gamma_{\xi}$ of the point $\xi$ acts discretely on $\widetilde{M}$ and the space $\widehat{W}_{\xi}$ is homeomorphic to the quotient of $\widetilde{M}$ by $\Gamma_{\xi}$. Put on each $\widehat{W}_{\xi}$ the smooth structure and the metric inherited from $\widetilde{M}$. The manifold $\widehat{W}_{\xi}$ and its metric vary continuously on $X_{M}$. The collection of all $\widehat{W}_{\xi}, \xi \in \widehat{M}$, form a continuous lamination $\widehat{\mathcal{W}}$ of $X_{M}$ with leaves which are manifolds locally modeled on $\widetilde{M}$. Denote by $\Delta^{\widehat{\mathcal{W}}}$ the laminated Laplace operator acting on functions which are smooth along the leaves of the lamination. A Borel measure $m$ on $X_{M}$ is called harmonic (with respect to $\widehat{\mathcal{W}}$ ) if it satisfies, for all $f$ for which it makes sense,

$$
\int \Delta^{\widehat{\mathcal{W}}} f d m=0
$$

Let $\widehat{m}$ be the $\Gamma$ invariant extension of a harmonic measure $m$ on $\widetilde{M} \times \widehat{M}$. There exists a finite measure $\widehat{\mathbf{m}}$ on $\widehat{M}([14])$ and, for $\widehat{\mathbf{m}}$-almost every $\xi$, a positive harmonic function $k_{\xi}(x)$ with $k_{\xi}\left(x_{0}\right)=1$ such that the measure $m$ can be written as

$$
\widehat{m}=k_{\xi}(x)(d x \times \widehat{\mathbf{m}}(d \xi)) .
$$

The set of harmonic probability measures is a weak* compact nonempty set of measures on $X_{M}$ ([14]). A harmonic probability measure $m$ is called ergodic if it is extremal among harmonic probability measures. As a corollary of the results in [28] and [33, Proposition 4.2], we have the following proposition.

Proposition 4.1. Let $(M, g)$ be a closed connected Riemannian manifold without focal points and $\ell^{2}=h$. Then, there exists an ergodic harmonic probability measure $m$ on $X_{M}$ such that $\Delta b_{\xi}=\ell$ for $\widehat{\mathbf{m}}$ almost all $\xi \in \widehat{M}$ (see below for definitions). 
Indeed, let $(M, g)$ be a closed connected Riemannian manifold. One can consider the Busemann compactification of $\widetilde{M}$ as follows. Fix a point $x_{0} \in \widetilde{M}$ as a reference point. For each point $x \in \widetilde{M}$, define a function $b_{x}(z)$ on $\widetilde{M}$ by

$$
b_{x}(z):=d(x, z)-d\left(x, x_{0}\right), \forall z \in \widetilde{M} .
$$

The assignment $x \mapsto b_{x}$ is continuous, one-to-one and takes values in a relatively compact set of functions for the topology of uniform convergence on compact subsets of $\widetilde{M}$. The Busemann compactification $\breve{M}$ of $\widetilde{M}$ is the closure of $\widetilde{M}$ for that topology. The space $\breve{M}$ is a compact separable space. The Busemann boundary $\breve{M}(\infty):=\breve{M} \backslash \widetilde{M}$ is compact ([29, Proposition 1]) and is made of 1-Lipschitz continuous functions $\breve{\xi}$ on $\widetilde{M}$ such that $\breve{\xi}\left(x_{0}\right)=0$. It is shown in 28. that if $\ell^{2}=h$, then there exists an ergodic harmonic probability measure $\breve{m}$ corresponding to the quotient of the space $\breve{M} \times \breve{M}$ by the diagonal action of $\Gamma$ such that $\Delta \breve{\xi}=\ell$ for $\breve{m}$ almost all $\breve{\xi} \in \breve{M}$. (Here, by $\Delta$, we mean the Laplacian in the distribution sense.) Note that in case $M$ has no focal points, there exists a homemorphism $\breve{\pi}: \breve{M} \mapsto \widetilde{M}$ (33. Proposition 4.2]) which satisfies $\breve{\xi}=b_{\breve{\pi} \breve{\xi}}$ for $\breve{\xi} \in \breve{M}$. Proposition 4.1 follows immediately by letting $m$ be the projection of $\breve{m}$ on $X_{M}$.

Proof of Proposition 2.3. Let $(M, g)$ be a closed connected rank one Riemannian manifold without focal points. Assume $\ell^{2}=h$. One can obtain a harmonic measure $m$ satisfying Proposition 4.1 by describing its $\Gamma$ invariant extension $\widehat{m}$ ([28, p. 720]). Set

$$
\widehat{m}_{t}:=\int_{\widetilde{M}} p(t, x, y) d y \frac{d x}{\operatorname{Vol} M},
$$

where $p(t, x, y), t \in \mathbb{R}_{+}, x, y \in \widetilde{M}$ is the heat kernel on $\widetilde{M}$. Then pick up $\widehat{m}$ as any ergodic decomposition of a limit point of $\frac{1}{T} \int_{0}^{T} \widehat{m}_{t} d t$. Its corresponding harmonic measure $m$ on $X_{M}$ satisfies Proposition 4.1 as required.

Note that the random Brownian path converges at $\widetilde{M}(\infty)$ by Theorem 3.12 . Hence we conclude from (4.1) that $\widehat{m}$ is actually pushed to be supported on $\widetilde{M} \times$ $\widetilde{M}(\infty)$. Thus, $m$ is one (and hence is the only one by Theorem 3.1) harmonic measure for the stable foliation. We also conclude from Theorem 3.1 that $m$ is fully supported on $S M$.

Now we have that $\widehat{\mathbf{m}}$ is fully supported on $\widetilde{M}(\infty)$. Moreover, by Proposition 4.1 , we have for $\widehat{\mathbf{m}}$-a.e. $\xi, \Delta b_{\xi}=\ell$. Recall from section 2.3 that the map $x \mapsto b_{\xi}(x)$ is of class $C^{2}$ and $\Delta_{x} b_{\xi}$ depends continuously on $\xi \in \widetilde{M}(\infty)$. We conclude that the Laplacian of the Busemann function $B$ on $S M$ is constant $\ell$. This shows $(\widetilde{M}, \widetilde{g})$ is asymptotically harmonic.

Remark 4.2. One can also show that any $m$ in Proposition 4.1 is fully supported on $S M$ without using the explicit description of the unique harmonic measure for the stable foliation in Theorem 3.1. Yet, we prefer to use it since it is independent of the assumption of $\ell^{2}=h$, has its own interest in the Dirichlet problem and might be useful in a further study of Martin boundary in the no focal points case. Here is one approach suggested by Zimmer. Any such $m$ also satisfies ([28])

$$
\nabla_{x} \ln k_{\xi}(x)=-\ell \nabla_{x} b_{\xi} \text {, for } \widehat{m} \text {-a.e. }(x, \xi) \in \widetilde{M} \times \widehat{M} \text {. }
$$


So $\widehat{\mathbf{m}}$-a.e. $\xi$ must belong to $\widetilde{M}(\infty)$ because of the nondifferentiability of $b_{\xi}$ at $\xi \in \widetilde{M}$ (cf. [33, Theorem 5.2]). Thus, $\widehat{m}$ is supported on $\widetilde{M} \times \widetilde{M}(\infty)$. By using the density of hyperbolic points in $\widetilde{M}(\infty)$ (Lemma 3.4), Lemma 3.5 and a similar argument as in [23, Lemma 4.1], one can obtain that each $\widehat{m}_{x}, x \in \widetilde{M}$, of the disintegration of $\widehat{m}$ is fully supported on $\widetilde{M}(\infty)$. It follows that $m$ is fully supported on $S M$.

\section{ACKNOWLEDGMENTS}

The first author was partially supported by NSF: DMS 0811127. The second author was partially supported by NSFC: 10901007 and China Scholarship Council; she would also like to thank the Department of Mathematics at the University of Notre Dame for their hospitality during her stay.

\section{REFERENCES}

[1] Michael T. Anderson and Richard Schoen, Positive harmonic functions on complete manifolds of negative curvature, Ann. of Math. (2) 121 (1985), no. 3, 429-461, DOI 10.2307/1971181. MR794369 (87a:58151)

[2] Werner Ballmann, On the Dirichlet problem at infinity for manifolds of nonpositive curvature, Forum Math. 1 (1989), no. 2, 201-213, DOI 10.1515/form.1989.1.201. MR990144 (90j:53059)

[3] Werner Ballmann, Lectures on spaces of nonpositive curvature, DMV Seminar, vol. 25, Birkhäuser Verlag, Basel, 1995. With an appendix by Misha Brin. MR.1377265 (97a:53053)

[4] Werner Ballmann and Patrick Eberlein, Fundamental groups of manifolds of nonpositive curvature, J. Differential Geom. 25 (1987), no. 1, 1-22. MR873453 (88b:53047)

[5] G. Besson, G. Courtois, and S. Gallot, Volume et entropie minimale des espaces localement symétriques (French), Invent. Math. 103 (1991), no. 2, 417-445, DOI 10.1007/BF01239520. MR 1085114 (92d:58027)

[6] G. Besson, G. Courtois, and S. Gallot, Entropies et rigidités des espaces localement symétriques de courbure strictement négative (French), Geom. Funct. Anal. 5 (1995), no. 5, 731-799, DOI 10.1007/BF01897050. MR1354289 (96i:58136)

[7] Yves Benoist, Patrick Foulon, and François Labourie, Flots d'Anosov à distributions stable et instable différentiables (French, with English summary), J. Amer. Math. Soc. 5 (1992), no. 1, 33-74, DOI 10.2307/2152750. MR1124979(93b:58112)

[8] P. Eberlein and B. O'Neill, Visibility manifolds, Pacific J. Math. 46 (1973), 45-109. MR0336648 (49 \#1421)

[9] Jost-Hinrich Eschenburg, Horospheres and the stable part of the geodesic flow, Math. Z. 153 (1977), no. 3, 237-251. MR0440605 (55 \#13479)

[10] Jost-Hinrich Eschenburg and John J. O'Sullivan, Growth of Jacobi fields and divergence of geodesics, Math. Z. 150 (1976), no. 3, 221-237. MR0418152 (54 \#6194)

[11] Patrick Foulon and François Labourie, Sur les variétés compactes asymptotiquement harmoniques (French), Invent. Math. 109 (1992), no. 1, 97-111, DOI 10.1007/BF01232020. MR.1168367 (93g:58114)

[12] A. Freire and R. Mañé, On the entropy of the geodesic flow in manifolds without conjugate points, Invent. Math. 69 (1982), no. 3, 375-392, DOI 10.1007/BF01389360. MR679763 (84d:58063)

[13] Harry Furstenberg, Boundary theory and stochastic processes on homogeneous spaces, Harmonic analysis on homogeneous spaces (Proc. Sympos. Pure Math., Vol. XXVI, Williams Coll., Williamstown, Mass., 1972), Amer. Math. Soc., Providence, R.I., 1973, pp. 193-229. MR0352328 (50 \#4815)

[14] Lucy Garnett, Foliations, the ergodic theorem and Brownian motion, J. Funct. Anal. 51 (1983), no. 3, 285-311, DOI 10.1016/0022-1236(83)90015-0. MR703080 (84j:58099)

[15] M. S. Goto, The cone topology on a manifold without focal points, J. Differential Geom. 14 (1979), no. 4, 595-598 (1981). MR600615(82d:53024)

[16] Y. Guivarc'h, Sur la loi des grands nombres et le rayon spectral d'une marche aléatoire (French, with English summary), Conference on Random Walks (Kleebach, 1979), Astérisque, vol. 74, Soc. Math. France, Paris, 1980, pp. 47-98, 3. MR588157 (82g:60016) 
[17] Robert Gulliver, On the variety of manifolds without conjugate points, Trans. Amer. Math. Soc. 210 (1975), 185-201. MR0383294 (52 \#4175)

[18] Sigurđur Helgason, Differential geometry and symmetric spaces, Pure and Applied Mathematics, Vol. XII, Academic Press, New York, 1962. MR.0145455 (26 \#2986)

[19] Donal Hurley, Ergodicity of the geodesic flow on rank one manifolds without focal points, Proc. Roy. Irish Acad. Sect. A 86 (1986), no. 1, 19-30. MR865098 (88a:58161)

[20] V. A. Kalmanovich, Brownian motion and harmonic functions on covering manifolds. An entropic approach (Russian), Dokl. Akad. Nauk SSSR 288 (1986), no. 5, 1045-1049. MR852647 (88k:58163)

[21] F. I. Karpelevič, The geometry of geodesics and the eigenfunctions of the Beltrami-Laplace operator on symmetric spaces, Trans. Moscow Math. Soc. 1965 (1967), 51-199. Amer. Math. Soc., Providence, R.I., 1967. MR.0231321 (37 \#6876)

[22] Anatole Katok, Four applications of conformal equivalence to geometry and dynamics, Ergodic Theory Dynam. Systems $\mathbf{8}^{*}$ (1988), Charles Conley Memorial Issue, 139-152, DOI 10.1017/S0143385700009391. MR967635(89m:58165)

[23] G. Knieper, On the asymptotic geometry of nonpositively curved manifolds, Geom. Funct. Anal. 7 (1997), no. 4, 755-782, DOI 10.1007/s000390050025. MR1465601 (98h:53055)

[24] Gerhard Knieper, New results on noncompact harmonic manifolds, Comment. Math. Helv. 87 (2012), no. 3, 669-703, DOI 10.4171/CMH/265. MR2980523

[25] F. Ledrappier, Harmonic measures and Bowen-Margulis measures, Israel J. Math. 71 (1990), no. 3, 275-287, DOI 10.1007/BF02773746. MR1088820(92a:58107)

[26] François Ledrappier, A heat kernel characterization of asymptotic harmonicity, Proc. Amer. Math. Soc. 118 (1993), no. 3, 1001-1004, DOI 10.2307/2160153. MR1137226 (93i:58146)

[27] François Ledrappier, Applications of dynamics to compact manifolds of negative curvature, 2 (Zürich, 1994), Birkhäuser, Basel, 1995, pp. 1195-1202. MR,1404020 (97e:58178)

[28] François Ledrappier, Linear drift and entropy for regular covers, Geom. Funct. Anal. 20 (2010), no. 3, 710-725, DOI 10.1007/s00039-010-0080-9. MR2720229 (2011i:58060)

[29] François Ledrappier and Xiaodong Wang, An integral formula for the volume entropy with applications to rigidity, J. Differential Geom. 85 (2010), no. 3, 461-477. MR2739810 (2012a:53068)

[30] Terry Lyons and Dennis Sullivan, Function theory, random paths and covering spaces, J. Differential Geom. 19 (1984), no. 2, 299-323. MR755228 (86b:58130)

[31] John J. O'Sullivan, Riemannian manifolds without focal points, J. Differential Geometry 11 (1976), no. 3, 321-333. MR0431036 (55 \#4038)

[32] J. Watkins, The higher rank rigidity theorem for manifolds with no focal points, Geom. Dedicata 164 (2013), 319-349. MR.3054631

[33] Andrew M. Zimmer, Compact asymptotically harmonic manifolds, J. Mod. Dyn. 6 (2012), no. 3, 377-403, DOI 10.3934/jmd.2012.6.377. MR2988813

Department of Mathematics, University of Notre Dame, Notre Dame, Indiana 465564618

E-mail address: fledrapp@nd.edu

LMam, School of Mathematical Sciences, Peking University, Beijing 100871, PeoPLE'S REPUBlic OF ChinA

E-mail address: 1shu@math.pku.edu.cn 